\title{
Heterogeneity in memory training improvement among older adults: A latent class analysis
}

\author{
Yana Fandakova ${ }^{1}$, Yee Lee Shing ${ }^{1,2}$, and Ulman Lindenberger ${ }^{1}$ \\ ${ }^{1}$ Max Planck Institute for Human Development, Center for Lifespan Psychology, Berlin, \\ Germany \\ ${ }^{2}$ Institute of Psychology, Humboldt-Universität zu Berlin, Berlin, Germany
}

\begin{abstract}
This study investigated the extent to which older adults' associative memory functioning can be modified through instruction and practice based on individuals' memory status. Here 42 younger adults and 42 older adults performed four tasks that measured strategic and binding aspects of memory. With latent class analysis, two classes of older adults were identified. The first class showed higher memory functioning similar to younger adults, while the second class was characterised by lower memory functioning. A subsequent analysis examined whether the high- and low-performing older adults differ in patterns of gain from receiving instruction and practice on a mnemonic strategy. The results revealed that high-performing older adults, similar to younger adults, showed higher associative memory performance under explicit intentional encoding instruction and after extensive practice of the strategy. In contrast, low-performing older adults benefited more from directed instruction of the strategy. The results are discussed in relation to individual differences in the functional status of mechanisms underlying memory functioning, and how these differences may lead to compensation or magnification of training gain. The present findings highlight the importance of considering differential memory processes to develop specific training paradigms that target the processes that show the most prominent decline.
\end{abstract}

Keywords: Ageing; Episodic memory; Individual differences; Training; Plasticity.

Episodic memory, the ability to remember past events that are situated in time and place (Tulving, 1972), undergoes profound decline with advancing adult age (Rönnlund, Nyberg, Backman, \& Nilsson, 2005; Singer, Verhaeghen, Ghisletta, Lindenberger, \& Baltes, 2003). Older adults show difficulties in remembering associative information (Old \& Naveh-Benjamin, 2008) and in monitoring source information (Henkel, Johnson, \& De Leonardis, 1998; Johnson, Hashtroudi, \& Lindsay, 1993; Spencer \& Raz, 1995). They also engage less in effective strategies such as clustering or elaborating information during encoding and retrieval (Cohn, Emrich, \& Moscovitch, 2008; Dunlosky \& Hertzog, 1998; Naveh-Benjamin, Brav, \& Levy, 2007), and show decline in recollection of specific contextual details during retrieval of episodic information (Jacoby, 1999; Jacoby \& Hay, 1998). The goal of the present study was to examine the extent to which older adults' associative memory functioning can be modified through instruction and extensive practice based on

Address correspondence to: Yee Lee Shing, Center for Lifespan Psychology, Max Planck Institute for Human Development, Lentzeallee 94, 14195 Berlin, Germany. E-mail: yshing@mpib-berlin.mpg.de

This study was conducted within the project "Cognitive and Neuronal Dynamics of Memory across the Lifespan (CONMEM)" at the Center for Lifespan Psychology, Max Planck Institute for Human Development. The study was conducted in partial fulfilment of the doctoral dissertation of the first author, who expresses gratitude to the support of the International Max Planck Research School on the Life Course (IMPRS LIFE). We give special thanks to Naftali Raz, Myriam Sander, Florian Schmiedek, Manuel Voelkle, and Markus Werkle-Bergner for valuable discussions. 
individuals' memory status. Specifically we identified individuals with high and low memory functioning based on four different tasks. We then related individuals' memory functional status to their gains in associative memory through mnemonic strategy instruction and practice.

\section{INDIVIDUAL DIFFERENCES IN EPISODIC MEMORY PERFORMANCE}

In addition to mean decline in episodic memory functioning, individual differences in episodic memory increase from early to late adulthood (de Frias, Lövdén, Lindenberger, \& Nilsson, 2007). Longitudinal investigations of memory functioning document reliable individual differences in the rates of change in old age (Christensen et al., 1999; de Frias et al., 2007; Hultsch, Hertzog, Dixon, \& Small, 1998; Lindenberger \& Ghisletta, 2009; Lövdén et al., 2004). For example, de Frias et al. (2007) found that, in addition to a decrease in mean performance, inter-individual differences in change for an episodic recall measure tend to increase in old age. Similarly, Lindenberger and Ghisletta (2009) showed that for older adults (ages 70 to 103 years at first measurement) change in memory for text and associative information across 13 years was associated with substantial degree of inter-individual differences in linear change across time.

On the neuronal level, brain regions contributing to episodic memory performance, in particular the prefrontal cortex (PFC) and medial temporal lobe (MTL; Simons \& Spiers, 2003), undergo remarkable structural and functional changes with age, both in mean change and inter-individual variability (Cabeza, Anderson, Locantore, \& McIntosh, 2002; Duverne, Motamedinia, \& Rugg, 2009; Fjell et al., 2009; Raz, Ghisletta, Rodrigue, Kennedy, \& Lindenberger, 2010). For instance, Raz et al. (2005) reported significant inter-individual differences in rate of change for lateral PFC $(d=.38)$ and the hippocampus $(d=.34)$ over the course of 5 years. This study also revealed a strong correlation $(r=.70)$ between grey matter volume loss in the hippocampus and white matter loss in PFC, indicating that agerelated structural changes in PFC and MTL regions are interdependent and may affect the functioning of episodic memory in a coordinated manner that differs substantially across individuals. Accordingly, a recent functional neuroima- ging study by Düzel, Schütze, Yonelinas, and Heinze (2011) revealed that, for older adults, less deviation from younger adults in terms of neural activation and structural integrity can be viewed as an index of successful ageing, as it is functionally related to intact recollection, a hallmark of episodic memory.

Taken together, behavioural and neuronal findings suggest that there is substantial inter-individual variability in the mean level of memory performance and in the rate of memory decline across elderly people. There is growing interest in the ageing field to develop training procedures that target memory functioning (cf. Noack, Lövdén, Schmiedek, \& Lindenberger, 2009). However, little is known about whether and how individual differences in memory functioning are related to gain in memory performance induced by instruction and training. Understanding the relationship between heterogeneity in memory functioning and training benefit in old age has important implications for episodic memory interventions as it can help to guide the design of training programmes that better target groups of individuals with different cognitive status (Lindenberger et al., 2008).

\section{INDIVIDUAL DIFFERENCES IN MEMORY TRAINING GAIN}

Plasticity denotes the capacity for change in the functional range of cognitive performance of an individual (Lövdén, Bäckman, Lindenberger, Schaefer, \& Schmiedek, 2010; see also Baltes, 1987; Kliegl \& Baltes, 1987). Generally, episodic memory performance in old age can be improved through appropriate instruction and training (Brehmer, Li, Müller, von Oertzen, \& Lindenberger, 2007; Noack et al., 2009; Verhaeghen, Marcoen, \& Goossens, 1992). However, older adults differ markedly in how much they benefit from cognitive training. The association between training benefits and cognitive ability is often captured by one of two competing models, the magnification model and the compensation model. The magnification model postulates that individuals who show higher performance on cognitive ability measures and demonstrate higher baseline performance in memory training programmes subsequently gain more from instruction and practice of mnemonic strategies (Bissig \& Lustig, 2007; Kliegl, Smith, \& Baltes, 1990; Verhaeghen \& 
Marcoen, 1996). In contrast, the compensation model postulates that the amount of gain through mnemonic instruction and practice is negatively correlated with baseline performance as well as with cognitive ability measures (Lövdén, Brehmer, $\mathrm{Li}, \&$ Lindenberger, 2012). In regard to this longstanding debate in the ageing literature, a more fine-grained examination of the mechanisms underlying the ability to benefit from memory interventions might be especially informative. An important feature that training paradigms need to incorporate is adapting training conditions to individuals' functional range. Lövdén, Bäckman, Lindenberger, Schaefer, and Schmiedek (2010) recently postulated that the driving force of plastic alterations is the prolonged mismatch between functional capacity and experienced demands. Hence, given the high degree of variability in memory performance observed among older adults, it is conceivable that varying training manipulations might have differential effects for individuals of the same age. Depending on differences in the functional status of the mechanisms underlying episodic memory, individuals may react better or worse to certain training procedures. This notion remains to be tested empirically.

The goal of the present study was to shed light on this issue by investigating the relationship between individual differences in episodic memory functioning and benefits from memory instruction and practice among older adults. A number of recent studies have suggested that high-performing older adults show behavioural performance and neuronal activation patterns that are similar to those of younger adults (e.g., Cappell, Gmeindl, \& Reuter-Lorenz, 2010; Duverne et al., 2009; Düzel et al., 2011; Nagel et al., 2009, 2011; SchneiderGarces et al., 2010). Hence, we hypothesised that high-performing older adults may show greater benefits from manipulations that are also highly beneficial for their younger counterparts (Buckner, 2004). In particular, younger adults tend to spontaneously use more effective encoding and retrieval strategies (Dunlosky \& Hertzog, 1998; Kausler, 1994; Naveh-Benjamin et al., 2007). Hence we expected that high-functioning older adults would be able to self-initiate memory strategies in a manner similar to younger adults. Furthermore, providing younger adults with extensive practice on a mnemonic strategy improves their memory performance, reflecting the activation of available cognitive resources (Kliegl, Smith, \& Baltes, 1989). Therefore we hypothesised that extensive practice would bring higher benefit to older adults with more available cognitive resources that can be utilised during training. In contrast, providing more environmental support was expected to be particularly beneficial for lowperforming older adults with fewer available cognitive resources (Craik, 1986; Verhaeghen et al., 1992).

We used latent class analysis (LCA; Hagenaars \& McCutcheon, 2002; Nagin, 1999) to identify classes of older adults with high and low memory functioning (cf. Düzel et al., 2011), and examined differences in associative memory between these classes following the instruction and practice of a mnemonic strategy. By using a combination of external measures to define classes of older adults that differ in memory functioning, the present approach has an advantage over traditional methods of median split or extreme group comparisons, as high- and low-performing older adults are identified independently of the training paradigm of main interest. In addition, the classification is more reliable and valid than splits based on a single observed variable. For the present analysis we used the data from younger and older adults previously reported by Shing, Werkle-Bergner, $\mathrm{Li}$, and Lindenberger (2008). In this study participants were instructed to encode word pairs under different encoding conditions, including incidental and intentional encoding of the binding between the words, as well as mnemonic strategy instruction and extensive practice of the strategy. The original publication of the data focused exclusively on mean differences between the age groups. In contrast, this article investigates whether interindividual differences in episodic memory functioning assessed with independent measures can predict heterogeneity in training gain among older adults.

\section{METHOD}

\section{Participants}

The current sample constituted a subsample of the training study of Shing and colleagues (2008), consisting of 42 younger adults $(52 \%$ female, age range $20-25)$ and 42 older adults (50\% female, age rage $70-75)$. The two age groups differed with regard to measures of fluid (Digit Symbol Substitution Test; Wechsler, 1955) and crystallised intelligence (Spot-a-word; Lehrl, 1977). The results followed the typical pattern found in the ageing literature (Baltes, Lindenberger, \& 
Staudinger, 1998), namely decline of fluid intelligence, $t(82)=8.84, \quad p<.05$, (younger adults: $M=62.8, \quad S D=10.9 ;$ older adults: $M=43.4$, $S D=9.5$ ), and preservation or increase in crystallised intelligence, $t(82)=7.86, p<.05$, (younger adults: $M=20.6, S D=4.5$; older adults: $M=27.9$, $S D=4.1)$.

\section{External class indicators}

We used four tasks as class indicators in the present analysis. Based on previous studies, the Face-Name Task (Naveh-Benjamin, Guez, Kilb, \& Reedy, 2004) and the Local Recognition Task (Oberauer, 2003) were assumed to rely more on the binding mechanisms of episodic memory that are dependent on MTL functioning (e.g., Davachi, 2006). Degree of using a clustering strategy in a modified version of the California Verbal Learning Task (CVLT; Delis, Kramer, Kaplan, \& Ober, 1987) and a Sort-Recall Memory Task (e.g., Schneider, Kron, Hunnerkopf, \& Krajewski, 2004) were assumed to rely more strongly on the strategic aspects of episodic memory that are dependent on PFC functioning (e.g., Miller \& Cohen, 2001).

Face-name task. In this task participants were presented with a total of 48 face-name pairs under incidental learning conditions (for more details on this task see Naveh-Benjamin et al., 2009). Participants were subsequently tested for their memories for face, name, and the association of face and name. For the current analysis we used data from the association test, since this test required the binding of a face to a name into a cohesive representation (see also NavehBenjamin et al., 2004).

Local recognition task. In this task six different consonants appeared consecutively on six different positions on a computer screen for $600 \mathrm{~ms}$ (for details see Oberauer, 2003). Participants were instructed to try to memorise both the letter and the position at which it occurred. At test participants indicated whether the consonants appeared on the same or on a different position as during study. This task required the binding of letters to locations into coherent memory representations in order to perform well.

For both tasks the score computed for each participant was hit rate (i.e., correct recognition of a studied pair) minus false alarm rate (i.e., rearranged face-name or letter-location pair wrongly accepted as an old pair). The range of this score was between 0 and 1 .

Modified California Verbal Learning Task. In the study phase of this task the participants heard a list of 16 German nouns at a 3-second pace. In the recall phase the participants wrote down as many nouns as they could remember in 3 minutes. Immediately after the recall phase the participants heard the list again followed by a recall phase. This procedure was repeated five times in total. The degree of clustering during recall was measured by the Adjusted Ratio of Clustering (ARC; Roenker, Brown, \& Thompson, 1971). The ARC reflects the degree to which studied items were strategically organised during recall, with a score of 0 indicating no sorting by category, and 1 indicating that all items were combined in categories. For the present analysis the mean ARC score across the five list repetitions was used. The ARC score was correlated with the number of recalled words for older adults $(r=.68$, $p<.05)$, but not for younger adults $(r=.15$, $p>.05)$. This might be due to the fact that younger adults' performance in the last repetitions of the word list was at ceiling and was therefore not related to clustering scores. Accordingly, younger adults' recall performance in the first two lists of the task was significantly correlated with the ARC scores for these lists $(r=.48$, $p<.05)$.

Sort-recall memory task. This task utilised a touch-screen monitor. Before starting the task all participants were instructed and given practice in how to use the setup. During the study phase words appeared consecutively on different random locations of the screen at a 2-second pace. After the last word appeared, all 24 words that constituted the study list were displayed on the screen (at original location) for another 90 seconds. Participants were instructed to remember the words during this time period. As the 24 words were drawn from four different semantic categories, it was possible to cluster the words using a touch-screen pen. After the study phase participants performed an interpolated activity for 60 seconds. Subsequently, participants had up to 3 minutes to recall as many words from the list as possible. The task consisted of a total of three study lists, each followed by a recall phase. For both age groups the ARC scores at study and recall were correlated (younger adults: $r=.24$, 
$p<.05$; older adults: $r=.64, p<.05$ ). However, to be comparable to the modified CVLT task, for the present analysis we used the ARC score from the recall phase of the Sort-Recall Task. This ARC score was also correlated with performance (younger adults: $r=.77, p<.05$; older adults: $r=.94, p<.05$ ), reflecting that the more participants strategically clustered their recall of memory items, the better their performance would be.

\section{Training data}

Associative recognition paradigm. In this paradigm participants studied lists of German-German and German-Malay word pairs under different encoding instructions across several sessions. In the first session participants were instructed to study each word from the pair individually for an upcoming item recognition test. ${ }^{1}$ As they did not expect a pair test, the relation between the two words was encoded incidentally (termed incidental encoding). In the following session the participants were instructed to study the words as intact pairs for an upcoming pair recognition test. The relation between the words was hence encoded intentionally (termed intentional encoding). In the third session participants were instructed in the use of an elaborative imagery strategy to encode the pairs. They were first introduced to the main principles of the strategy, followed by concrete examples on how to apply the strategy to the word pairs. In two subsequent sessions the participants encoded the word pairs using the instructed strategy (termed post-instruction assessments). For the GermanMalay condition, the participants underwent extensive practice of the elaborative strategy with five further sessions. In each of these practice sessions the participants practised encoding the German-Malay word pairs using the imagery strategy. Participants were provided with feedback on how to improve the quality of their generated images (e.g., to be vivid and unique). In a subsequent final session (termed post-practice assessment) memory performance was assessed again.

\footnotetext{
${ }^{1}$ During the recognition phase of the training paradigm participants worked on both an item and a pair recognition test. However, only data from the pair recognition test was used in the present analysis, as successful performance in this test required recollection of the binding between the two words in order to successfully detect intact study pairs and reject rearranged pairs Detailed description of the results from the German-German condition and the item recognition test can be found in Shing et al. (2008).
}

At each of the assessments (except the postpractice assessment), each participant studied and was subsequently tested on one German-German and one German-Malay pair-recognition list. The list order was randomised across participants. At the post-practice assessment only German-Malay pair recognition was assessed. All word lists were composed of unique words only shown in one session.

At the beginning of each session the participants were given the corresponding encoding instruction as described above. During the encoding phase, lists of 45 German-German or German-Malay word pairs were presented sequentially on a computer screen. Presentation rate of the word pairs was set to 6 seconds for older adults and 3 seconds for younger adults in order to avoid possible ceiling effects in younger adults (for more details see Shing et al., 2008). After encoding of the word list participants performed an interpolated activity for 90 seconds. During the recognition phase, 60 words pairs were sequentially displayed on the screen and participants had to judge whether they had seen the word pairs during encoding. Half of the word pairs were intact pairs from the study list. From the remaining 30 pairs, 15 were new pairs consisting of two words that were not presented previously, and 15 were rearranged pairs composed of two words that were taken from different study pairs. Participants were given up to 5 seconds to respond. Each old/new judgement was followed by a confidence rating of participants' decision on a 3-point scale (for more details on the analysis of confidence judgments see Shing Werkle-Bergner, Li, \& Lindenberger, 2009).

At each of the assessments, memory performance was measured for each participant by subtracting the false alarm rate to the rearranged pairs from the hit rate (Pr-values; Snodgrass \& Corwin, 1988). In the present analysis we used only data from the German-Malay condition, since participants underwent extensive practice of a mnemonic strategy only for this condition. Word list order (German-Malay or German-German list studied first) did not affect memory performance and data across the two conditions were collapsed. There were no differences in performance between the two post-instruction assessments within both age groups. Therefore a mean score of the two assessments is used in the present analysis (termed post-instruction assessment). 


\section{Covariate measures}

Digit and animal sorting task. All participants performed both conditions of the task in a fixed order (Kray \& Lindenberger, 2000). The digit sorting condition was performed first, followed by the animal sorting condition. Each condition consisted of three practice trials and 15 task trails. In each trial a list of digits (digit sorting condition) or animals (animal sorting condition) ranging from three to eight items was presented auditorily to the participants. In the digit sorting condition participants were asked to repeat the digits by sorting them according to numerosity. In the animal sorting condition participants had to repeat the animals by sorting them in order of their body-size differences (for more details see Kray \& Lindenberger, 2000). The mean number of correctly sorted lists across both conditions was used as a measure of working memory in the present analysis.

Personal beliefs about memory instrument $(P B M I)$. The PBMI measures beliefs about one's own memory (Lineweaver \& Hertzog, 1998). It consists of three subscales: memory efficacy, change, and control beliefs. For each item participants in the present study were asked to provide an answer by making a graphic rating scale response between the two endpoints of the scale. Responses were measured in millimetres of distance from the lower endpoint of the scale, with higher values indicating higher scores on each of the subscales.

\section{Data analysis}

In the present analysis LCA was used to capture class membership indicated by the four continuous indicator measures. LCA is a latent variable modelling technique used to identify classes of observations characterised by similar values on class indicators (Hagenaars \& McCutcheon, 2002; Loken \& Molenaar, 2008). Similar to other latent variable models, LCA assumes that person's observed indicator values are caused by the person's value on a latent categorical variable. The data on the indicators are assumed to be sampled from a population that is composed of a mix of distributions, one for each of the classes, characterised by its own set of parameters. In the current study missing data were accommodated using Maximum Likelihood Estimation (Bollen \&
Curran, 2006) to estimate the LCA model parameters. Bayesian information criteria (BIC; Schwartz, 1978) and the Lo-Mendell-Rubin likelihood ratio test (LMR; Lo, Mendell, \& Rubin, 2001) were used to compare models with different number of classes and to decide for the model that explains the data most adequately and parsimoniously. The best-fit model generally shows lower BIC and small $p$-values associated with the LMR test. The entropy statistic $\mathbf{E}$ was used to judge the accuracy of the assignment of persons to classes. It ranges from 0 to 1 , with higher values indicating higher classification utility. In a final step we examined the relationship between class membership and external variables in order to determine the validity of the obtained solution and to better characterise memory performance and plasticity in the two classes.

The data on the four indicator variables were submitted to the LCA as implemented in Mplus (Version 5.2; Muthén \& Muthén, 1998-2010) without any information with regard to age group. Four models were tested: one-, two-, three-, and four-class solutions. For all models unique means and variances were estimated for each class. The covariances of the external indicators were kept the same across classes. Existing evidence suggests that correlations among cognitive tasks increase in old age (e.g., Baltes \& Lindenberger, 1997; Lindenberger \& Ghisletta, 2009). However, given our interest to identify patterns of mean level differences in the indicator measures, we constrained their covariances to be equal across classes. Given that no distinction was made between age groups we expected that a two-class solution would fit the data best, reflecting previously reported age differences in episodic memory (Shing et al., 2008).

\section{RESULTS}

\section{Preliminary analysis}

The current study has a relatively small sample size for the application of LCA. Therefore special attention was given to the screening of the variables used in the analysis. Each of the indicator variables was examined for outliers at $p<.001$ (two-tailed test). The data of two younger and two older adults in the Face-Name Task were excluded from analysis due to an excessive amount of missing trials. No outliers were found for any of the measures. To examine distribution 
normality, the skewness and kurtosis values for each of the four indicator measures (Table 1) were tested against zero by using the $z$-distribution (Kline, 2005; Tabachnik \& Fidell, 2006). Problematic values are indexed by $z$-scores exceeding $|3|(p<.01$, two-tailed test $)$. No such cases were detected. An alpha level of .05 was used for all statistical tests, and effect sizes of reliable effects were indicated by the intraclass correlation coefficient $\left(p_{1}\right)$ or Cohen's $d$ (Fern \& Monroe, 1996).

\section{Latent class analysis}

As predicted, the two-class solution showed best fit to the data, as indicated by the BIC and LMR tests: one-class solution: number of parameters $=10$, Loglikelihood $(\mathrm{LL})=16.28, \mathrm{BIC}=11.75$; twoclass solution: number of parameters $=19$, $\mathrm{LL}=47.23, \mathrm{BIC}=-10.27, p \mathrm{LMR}=.00$; threeclass solution: number of parameters $=28$, $\mathrm{LL}=66.06, \mathrm{BIC}=-8.05, p \mathrm{LMR}=.13$; four-class solution: number of parameters $=37, \mathrm{LL}=81.03$, $\mathrm{BIC}=1.88, p \mathrm{LMR}=.37$. The first class (referred as high-performance class) was characterised by higher values than the second class (referred as low-performance class) on all indicator variables (Figure 1). The class probability was $p=.99$ and $p=.92$ for the high- and low-performance classes, respectively. The entropy statistic equalled $\mathrm{E}=.86$.

When examining the distribution of younger and older adults, most of the younger adults $(N=38)$ showed high values on the indicator variables and were classified in the high-performance class. The remaining younger adults $(N=4)$ were classified as belonging to the lowperformance class, primarily because of low scores on the Local Recognition Task. On the contrary, most of the older adults were classified in the low-performance class $(N=26)$. Nevertheless, a subgroup of older adults $(N=16)$ was classified as belonging to the high-performance class. The high-performing older adults showed better performance than the low-performing older adults on the Local Recognition and Sort-Recall Tasks (Figure 1). Within the highperformance group the older adults differed from the younger adults on all indicator variables except the Local Recognition Task.

\section{Training data}

With the data from the associative recognition paradigm (Figure 2), we examined the effects of performance class (between-person factor: high vs low) and encoding condition (within-person factor: incidental encoding vs intentional encoding vs post-instruction assessment vs post-practice assessment). A two-way analysis of variance (ANOVA) revealed that there was a significant effect of encoding condition, $F(3,195)=48.69, p<.05$, $p_{\mathrm{I}}=.65$, class, $F(1,65)=13.10, p<.05, p_{\mathrm{I}}=.41$, as well as a significant encoding condition $\times$ class interaction, $F(3,195)=5.74, p<.05, p_{\mathrm{I}}=.28$. These results indicated that the two classes differed with respect to how their memory performance changed across the different encoding instructions in the study.

To validate the results of the LCA, we first examined whether high-performing younger and older adults differed in their memory performance (Table 2). For this we analysed the effect of encoding condition and age on memory performance within the high-performance class. There was a significant effect of encoding condition, $F(3,114)=45.40, p<.05, p_{1}=.74$, but no effect of

TABLE 1

Skewness and kurtosis of the four external indicator measures

\begin{tabular}{lccrr}
\hline & Face-name & Local recognition & Sort-recall & CVLT \\
\hline Younger adults & & & & \\
Number of cases & 40 & 42 & 42 & 42 \\
Skewness (SE) & $0.11(0.37)$ & $-0.24(0.37)$ & $-0.76(0.37)$ & $1.99(0.72)$ \\
Kurtosis (SE) & $0.29(0.73)$ & $-1.03(0.72)$ & & $-0.46(0.37)$ \\
Older adults & & & 42 & $-0.06(0.72)$ \\
Number of cases & 40 & 42 & $-0.01(0.37)$ & 42 \\
Skewness (SE) & $0.07(0.37)$ & $0.67(0.37)$ & $-0.46(0.72)$ & $0.27(0.37)$ \\
Kurtosis (SE) & $0.55(0.73)$ & $-0.19(0.72)$ & $-1.41(0.72)$ \\
\hline
\end{tabular}


age, $F(1,38)=2.70, p>.05, p_{1}=.26$, nor encoding condition $\times$ age interaction, $F(3,114)=1.30$, $p>.05, p_{\mathrm{I}}=.18^{2}$ Taken together, younger and older adults in the high-performing class showed similar patterns of change across different encoding instructions. We did not perform the same validation with the low-performance class due to the limited number of younger adults in this group.

Next we examined the effect of performance class and encoding condition within the group of older adults. With this analysis we addressed the main question of the present analysis, namely to what extent belonging to a class of high or low episodic memory functioning is related to differences in memory training improvement among older adults. The two-way ANOVA revealed a significant effect of encoding condition, $F(3,108)=26.81, p<.05, p_{\mathrm{I}}=.65$, and class, $F(1,36)=4.37, p<.05, p_{\mathrm{I}}=.33$. Moreover, the interaction between encoding condition and class was significant, $F(3,108)=5.13, p<.05, p_{\mathrm{I}}=.35$, indicating that high-performing and low-performing older adults differed in how their performance changed across different encoding conditions (Table 2). Post-hoc comparisons showed that while high and low performers did not differ under incidental encoding instruction, $t(40)=-0.20, p>.05, d=0.06$, high performers showed higher memory performance under intentional encoding instruction, $t(40)=2.09, p<.05$, $d=0.66$. In contrast, differences between the two

\footnotetext{
${ }^{2}$ We performed a set of control analyses to examine the possibility that absence of differences between high-performing younger and older adults in the training paradigm might be driven primarily by their performance under intentional encoding instructions. Specifically we examined performance in the associative recognition paradigm while excluding the intentional encoding condition. The resulting ANOVA with effects of age group (between-person factor: younger vs older) and encoding condition (within-person factor: incidental encoding vs post-instruction assessment vs post-practice assessment) revealed a significant effect of encoding condition, $F(2,76)=63.63, p<.05$, and age, $F(1,38)=5.14, p<.05$, but no significant age $\times$ encoding condition interaction, $F(2$, 76) $=0.04, p>.05$. Post-hoc comparisons indicated that the age effect was driven mainly by differences at the postinstruction assessment $(F(1,52)=7.04, p<.05)$. Hence younger adults seemed to benefit more from the strategy instruction than the high-performing older adults. Based on our observation, this is possibly because younger adults generated more vivid and unique images with the mnemonic strategy. In contrast, the two age groups did not differ under the incidental encoding instruction, $F(1,51)=1.78, p>.05$, as well as at the post-practice assessment, $F(1,39)=1.31, p>.05$. Taken together our main findings remained valid, as there was no difference between high-performing older and younger adults under intentional and post-practice encoding conditions.
}

classes were absent at the post-instruction assessment, $t(40)=1.73, p>.05, d=0.54$. However, at the post-practice assessment the high-performers again showed superior memory performance, reflecting higher gain from the extensive practice of the strategy, $t(36)=2.44, p<.05, d=0.82$.

Finally, when we compared the older adults on other covariates, differences between the highand low-performance classes (Figure 3) were found for measures of working memory, $t(40)=4.56, p<.05, d=1.45$, processing speed, $t(40)=2.30, p<.05, d=0.72$, and vocabulary, $t(40)=3.02, p<.05, d=0.95$. Older adults from the two classes also differed with regard to their perceived control over memory (control subscale of the PBMI), $t(40)=3.25, p<.05, d=1.03$, with high-performing older adults reporting higher level of subjective control over memory in comparison to low-performing older adults.

\section{DISCUSSION}

Results from the present study revealed that heterogeneity in memory functioning among older adults is related to differences in benefit from a memory training intervention. In particular, highperforming and low-performing older adults demonstrated distinctive patterns of change across varying encoding conditions. Initially, older adults in the two classes did not differ in performance under incidental encoding instruction. However, when given instructions to encode the words together as a pair (intentional encoding condition), the high-performing older adults showed better performance compared to the low-performing older adults. After instruction of the imagery strategy, the differences between the two classes disappeared. Finally, differences in performance between the high-performing and low-performing older adults emerged again at the post-practice assessment, driven by increased performance of the high-performing older adults after extensive practice of the strategy. Taken together, these findings indicate that heterogeneity in episodic memory functioning leads to differential benefits from memory instruction and practice.

The present results are in agreement with previous studies showing that older adults can benefit from training of an effective memory strategy (Baltes \& Kliegl, 1992; Brehmer et al., 2007; Kliegl et al., 1989, 1990). They also confirm earlier longitudinal and cross-sectional findings of 


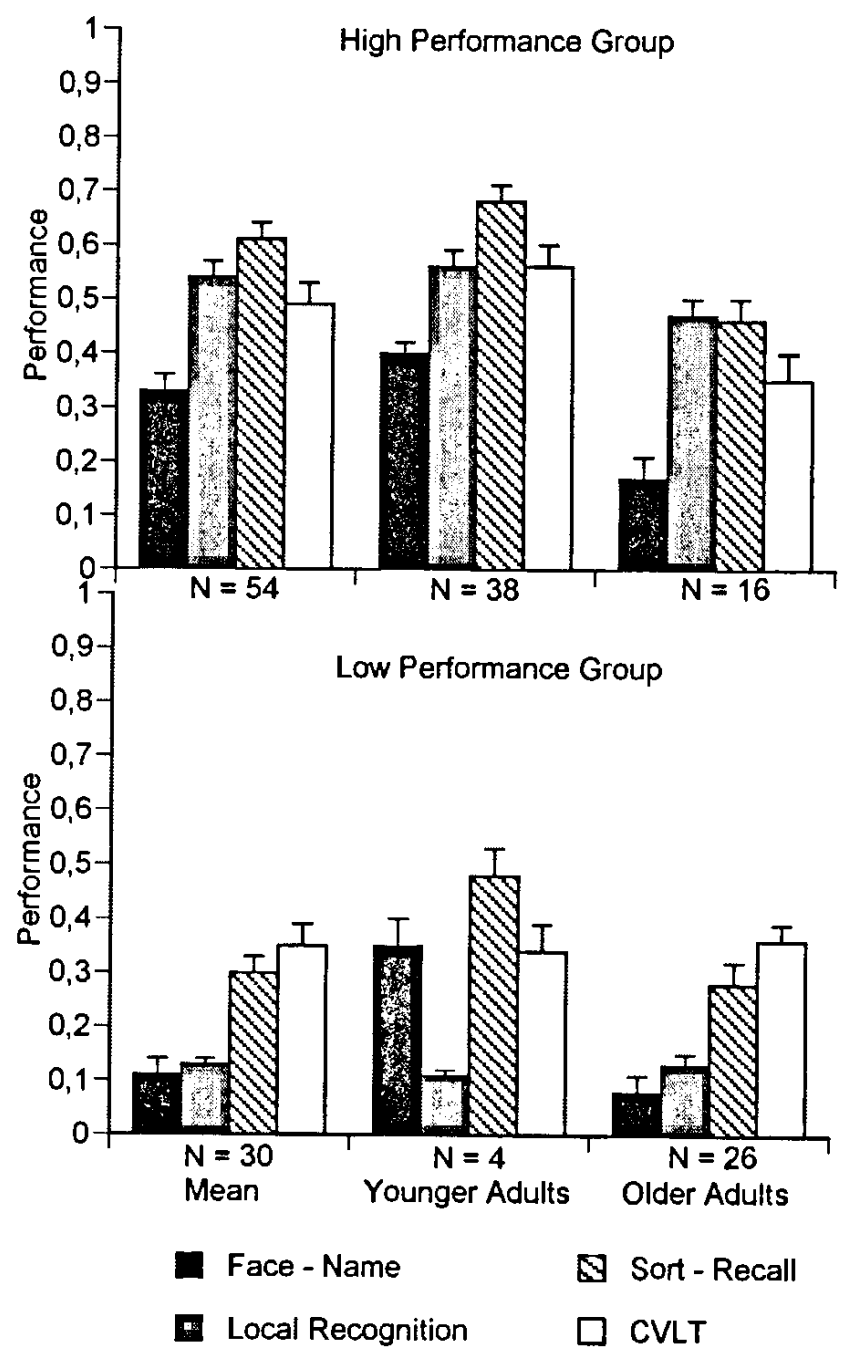

Figure 1. Mean performance on the external indicator measures for each class and for each age group within each class. Error bars represent standard error of the mean.

increased heterogeneity in old age (de Frias et al., 2007; Lindenberger \& Ghisletta, 2009). A novel aspect of the present research is piecing together these lines of evidence and pointing to mechanisms that may underlie heterogeneity in the ability to benefit from training interventions and improve memory performance in old age. In the following we interpret our findings in terms of the two-component framework of episodic memory development across the lifespan (Shing et al., 2008, 2010). The two-component framework postulates that older adults' difficulty in episodic memory is related to declines in both the strategic and associative components of episodic memory. The strategic component refers to control processes that aid and regulate memory functions at encoding and retrieval, whereas the associative component refers to mechanisms that bind different features of memory events into coherent representations. The framework, initially proposed to account for age differences in levels of episodic memory performance, may also account for individual differences in episodic memory functioning and plasticity. Specifically, the superior performance of the high-performing older adults under intentional encoding instruction in the present study might reflect their ability to make use of a relatively preserved strategic component to self-initiate and apply strategies for remembering the word pairs (see also Glisky, Rubin, \& Davidson, 2001). This interpretation is corroborated by the observation that high-performing older adults in the current study also reported to have more personal control over memory, in line with a preserved ability to self-initiate strategies to facilitate memory performance. Furthermore, while high and low performers did not differ after instruction of the imagery strategy, the high performers showed higher memory scores after extensive practice of the strategy. These results suggest that while low-performing older adults benefited 


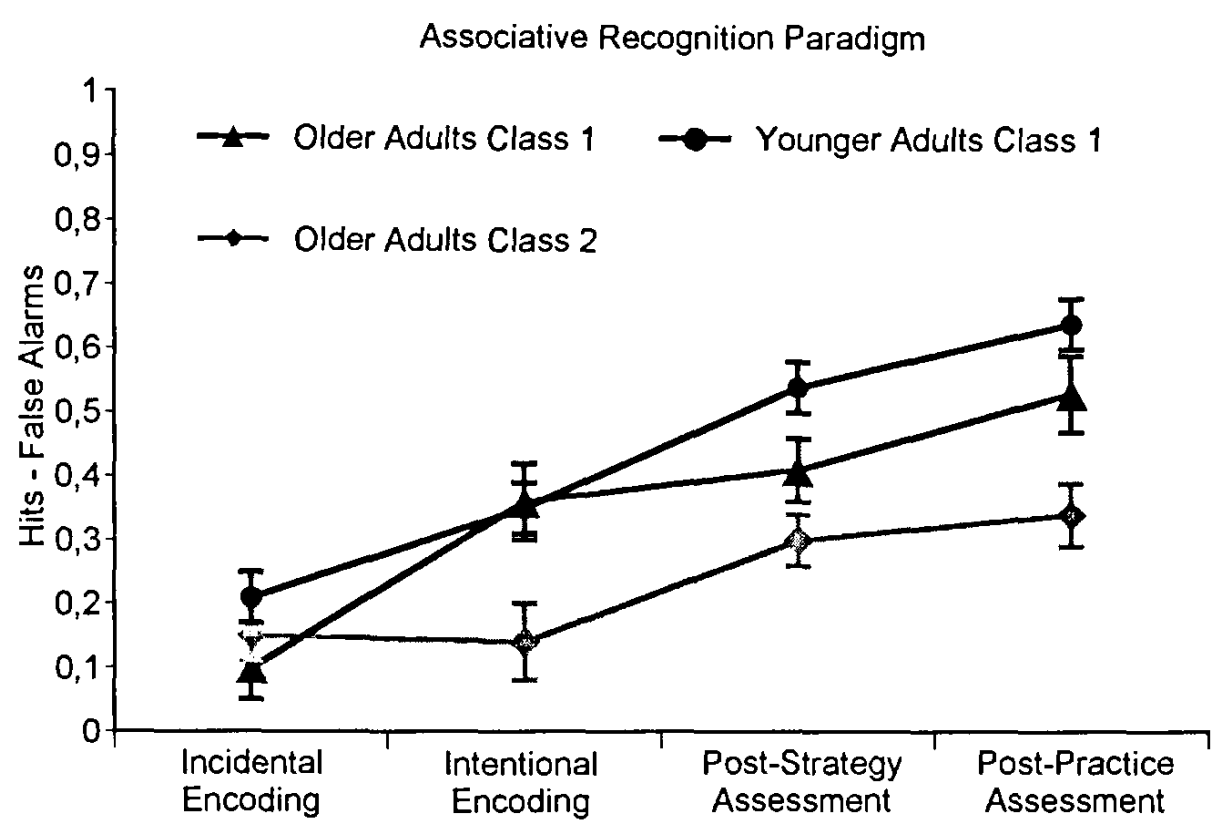

Figure 2. Differences between younger adults in the high-performance class, and older adults in the high-and low-performance classes for different the encoding conditions of the associative recognition paradigm. Error bars represent standard error of the mean.

from strategy instruction, only the high-performing older adults were able to benefit further from additional practice sessions, possibly reflecting their relatively preserved associative component (cf. Brehmer et al., 2007; Lövdén et al., 2012).

Craik (e.g., 1986) introduced the distinction between self-initiated processing and environmental support to capture individual and age-related differences in the need and ability to benefit from facilitating contexts. This distinction might also be related to the context of the two-component framework, as the degree to which older adults need and are able to benefit from environmental support may depend on the functional status of the strategic and associative memory components. For some older adults it might be sufficient to arrange the environment in a way that promotes opportunity to initiate intentional learning, for others a more directed instruction of a strategy might be needed, and for still others, any form of support may be insufficient. Extensive practice in applying a strategy, as implemented in the current training paradigm (Shing et al., 2008), may not be beneficial for older adults whose associative component is severely impaired. Different training procedures that directly target the associative component may be needed for these individuals. Importantly, the finding that both groups of older adults did not differ immediately after instruction of the strategy suggests that boosting memory performance in the course of a training programme might be achieved through different manipulations that correspond to individual differences in the functional status of the mechanisms supporting episodic memory (cf. Düzel et al., 2011). This observation is in line with the prediction of the

TABLE 2

Means (SD) of hit - false alarm rates across different encoding conditions

\begin{tabular}{lcccc}
\hline & Incidental encoding & Intentional encoding & Post-instruction assessment & Post-practice assessment \\
\hline $\begin{array}{l}\text { High-performance } \\
\text { Younger adults } \\
(N=38)\end{array}$ & $.21(0.23)$ & $.32(0.23)$ & $.52(0.17)$ & $.62(0.24)$ \\
$\begin{array}{l}\text { Older adults } \\
(N=16)\end{array}$ & $.12(0.18)$ & $.32(0.23)$ & $.38(0.19)$ & $.58(0.23)$ \\
$\begin{array}{l}\text { Low-performance } \\
\text { Younger adults }\end{array}$ & $.31(0.24)$ & $.32(0.11)$ & $.51(0.10)$ & $.62(0.24)$ \\
$\begin{array}{l}N=4) \\
\begin{array}{l}\text { Older adults } \\
(N=26)\end{array}\end{array}$ & $.13(0.19)$ & $.15(0.27)$ & $.29(0.17)$ & $.34(0.22)$ \\
\hline
\end{tabular}




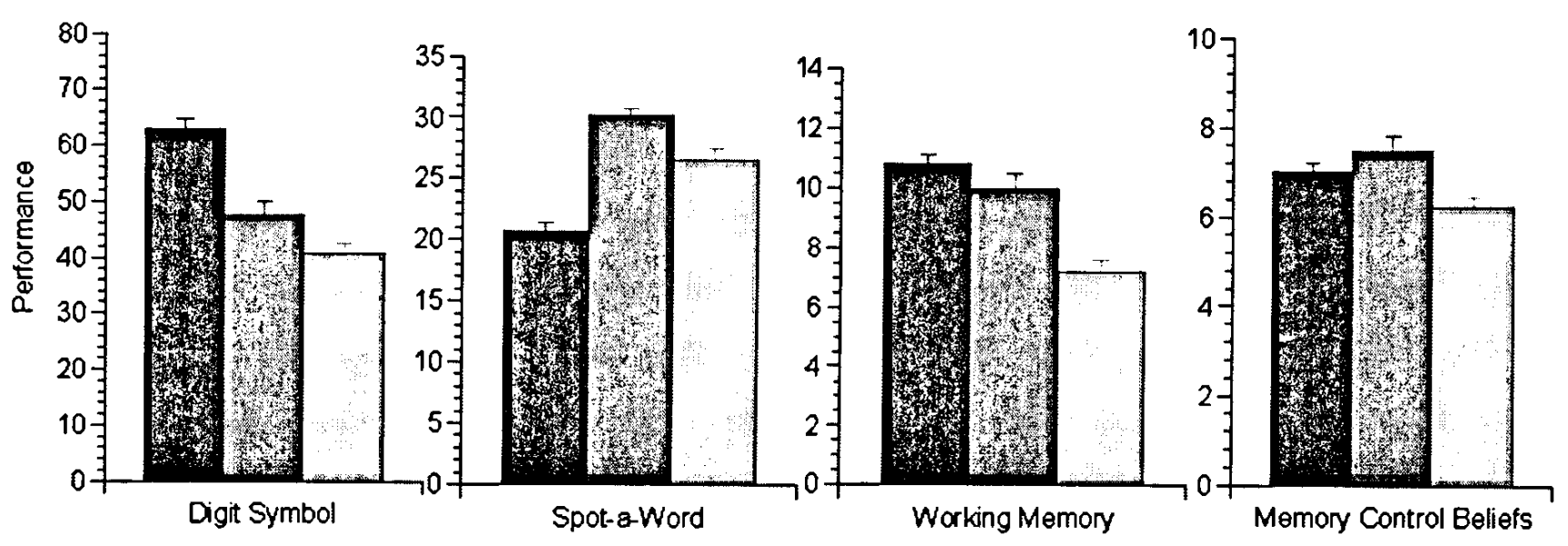

Figure 3. Differences between younger adults in the high-performance class, and older adults in the high- and low-performance classes for measures of processing speed (Digit Symbol), vocabulary (Spot-a-word), working memory (Digit and Animal Sorting Task), and perceived control over memory (PBMI, Control subscale). Error bars represent standard error of the mean.

compensation model. At the same time, the fact that the high-performing older adults showed higher performance after extensive practice of the mnemonic strategy clearly supports the magnification model. Taken together, our results indicate that the extent to which compensation or magnification applies depends very much on the match between memory training manipulations and functional status of individuals' memory system.

Confirmatory factor analysis (CFA) and latent class analysis (LCA) are formally related and highlight different aspects of the data (see also Loken \& Molenaar, 2008). Specifically, the latent categorical variable modelled in the present analyses represents individuals' likelihood of being a member of a latent class. Hence we may imagine that ageing individuals experience a continuous longitudinal progression of decline that is represented by a continuous shift of the probability of membership from high to low performing. In this sense LCA does not postulate the existence of separate entities. To illustrate this point we correlated older adults' performance in the training paradigm with their probability of belonging to the high-performing class. Under the intentional encoding condition, probability of belonging to the high-performing class correlated with performance $(\mathrm{r}=.34, \mathrm{p}<.05)$. In contrast, performance under the incidental encoding condition was not reliably related to the probability of belonging to the high-performing class $(\mathrm{r}=-.04, \mathrm{p}>.05)$. This finding is consistent with the absence of differences between high and low performers under the incidental encoding condition (see Figure 2).
As a statistical tool, LCA is superior over median split or extreme group designs (e.g., Maxwell \& Delaney, 1993). Just like CFA, which is a variable-centred SEM technique, LCA, as a person-oriented SEM technique, uses latent variables. Hence LCA focuses on common variance and explicitly accounts for data unreliability (i.e., measurement error). The latent classes identified by LCA are optimal in the sense that they maximise the probability of the data under the model. In contrast, median split or extreme group procedures are based on more or less arbitrary decisions; that is, on decisions that are not optimal on the basis of some statistical criterion.

Finally we note that the relatively small sample sizes of the present study might have prevented the detection of smaller classes of individuals. For instance, based on the two-component framework of episodic memory, one would expect that high and low performers could be further teased apart by their functioning on the strategic and associative components (Shing et al., 2010). This is especially relevant given that some forms of pathological ageing like Alzheimer's disease might affect the associative component to a greater degree than the strategic component (Bäckman, Jones, Berger, Laukka, \& Small, 2005; Buckner, 2004; Düzel et al., 2011). Future studies should combine behavioural and neuroimaging approaches in a longitudinal context to further delineate the relationship between individual differences in functional status and training improvements in memory performance across the lifespan.

Manuscript received 18 November 2010 Manuscript accepted 11 April 2012 First published online 30 May 2012 


\section{REFERENCES}

Bäckman, L., Jones, S., Berger, A. K., Laukka, E. J., \& Small, B. J. (2005). Cognitive impairment in preclinical Alzheimer's disease: A meta-analysis. Neuropsychology, 19, 520-531. doi: 10.1037/0894-4105.19. 4.520

Baltes, P. B. (1987). Theoretical propositions of lifespan developmental psychology: On the dynamics between growth and decline. Developmental Psychology, 23, 611-626. doi: 10.137/0012-1649.23. 5.611

Baltes, P. B., \& Kliegl, R. (1992). Further testing of limits of cognitive plasticity: Negative agedifferences in a mnemonic skill are robust. Developmental Psychology, 28, 121-125. doi:10.1037/00121649.28.1.121

Baltes, P. B., \& Lindenberger, U. (1997). Emergence of a powerful connection between sensory and cognitive functions across the adult life span: A new window to the study of cognitive ageing? Psychology and Ageing, 12, 12-21. doi:10.1037/0882-7974. 12.1.12

Baltes, P. B., Lindenberger, U., \& Staudinger, U. M. (1998). Lifespan theory in developmental psychology. In W. Damon \& R. M. Lerner (Eds.), Handbook of child psychology: Vol. 1. Theoretical models of human development (5th ed., pp. 10291143): New York: Wiley.

Bissig, D., \& Lustig, C. (2007). Who benefits from memory training? Psychological Science, 18, 720-726. doi: 10.1111/j.1467-9280.2007.01966

Bollen, K. A., \& Curran, P. J. (2006). Latent curve models: A structural equation perspective. Hoboken, NJ: John Wiley \& Sons.

Brehmer, Y., Li, S. C., Müller, V., von Oertzen, T., \& Lindenberger, U. (2007). Memory plasticity across the lifespan: Uncovering children's latent potential. Developmental Psychology, 43, 465-478. doi: 10.1037/0012-1649.43.2.465

Buckner, R. L. (2004). Memory and executive function in ageing and $\mathrm{AD}$ : Multiple factors that cause decline and reserve factors that compensate. Neuron, 44, 195-208. doi: 10.1016/j.neuron.2004.09.006

Cabeza, R., Anderson, N. D., Locantore, J. K., \& McIntosh, A. R. (2002). Ageing gracefully: Compensatory brain activity in high-performing older adults. Neuroimage, I7, 1394-1402. doi: S1053811 902912802

Capell, K. A., Gmeindl, L., \& Reuter-Lorenz P. A. (2010). Age differences in prefrontal recruitment during verbal working memory maintenance depend on memory load. Cortex, 46, 462-473. doi:10.1016/ j.cortex.2009.11.009

Christensen, H., Mackinnon, A. J., Korten, A. E., Jorm, A. F., Henderson, A. S., Jacomb, P. et al.. (1999). An analysis of diversity in the cognitive performance of elderly community dwellers: Individual differences in change scores as a function of age. Psychology and Ageing, 14, 365-379. doi: 10.1037/0882-7974. 14.3.365

Cohn, M., Emrich, S. M., \& Moscovitch, M. (2008). Age-related deficits in associative memory: The influence of impaired strategic retrieval. Psychology and Ageing, 23, 93-103. doi: 10.1037/0882-7974. 23.1.93

Craik, F. I. (1986). A functional account of age differences in memory. In F. Klix \& H. Hagendorf (Eds.), Human memory and capabilities: Mechanisms and performances (pp. 409-422). Amsterdam: Elsevier.

Davachi, L. (2006). Item, context and relational episodic encoding in humans. Current Opinion in Neurobiology, 16, 693-700. doi: 10.1016/j.conb.2006.10.012

de Frias, C. M., Lovden, M., Lindenberger, U., \& Nilsson, L. G. (2007). Revisiting the dedifferentiation hypothesis with longitudinal multi-cohort data. Intelligence, 35, 381-392. doi: 10.1016/j.intell.2006.07.011

Delis, D. C., Kramer, J. H., Kaplan, E., \& Ober, B. A. (1987). The California Verbal Learning Test: Research edition. New York: Psychological Corporation.

Dunlosky, J., \& Hertzog, C. (1998). Ageing and deficits in associative memory: What is the role of strategy production? Psychology and Ageing, 13, 597-607. doi: $10.1037 / 0882-7974.13 .4 .597$

Duverne, S., Motamedinia, S., \& Rugg, M. D. (2009). The relationship between ageing, performance, and the neural correlates of successful memory encoding. Cerebral Cortex, 19, 733-744. doi: 10.1093/ cercor/bhn122

Düzel, E., Schütze, H., Yonelinas, A. P., \& Heinze, HJ. (2011). Functional phenotyping of successful aging in long-term memory: Preserved performance in the absence of neural compensation. Hippocampus, 21, 803-814. doi: $10.1002 /$ hipo.20834

Fern, E. F., \& Monroe, K. B. (1996). Effect-size estimates: Issues and problems in interpretation. Journal of Consumer Research, 23, 89-105.

Fjell, A. M., Walhovd, K. B., Fennema-Notestine, C., McEvoy, L. K., Hagler, D. J., Holland, D. et al. (2009). One-year brain atrophy evident in healthy ageing. Journal of Neuroscience, 29, 15223-15231. doi: 10.1523/JNEUROSCI.3252-09.2009

Glisky, E. L., Rubin, S. R., \& Davidson, P. S. R. (2001). Source memory in older adults: An encoding or retrieval problem? Journal of Experimental Psychology: Learning, Memory, and Cognition, 27, 11311146. doi:10.1037/0278-7393.27.5.1131

Hagenaars, J. A., \& McCutcheon, A. L. (2002). Applied latent class analysis. New York: Cambridge University Press.

Henkel, L. A., Johnson, M. K., \& De Leonardis, D. M. (1998). Ageing and source monitoring: Cognitive processes and neuropsychological correlates. Journal of Experimental Psychology: General, 127, 251-268. doi: 10.1037/0096-3445.127.3.251

Hultsch, D. F., Hertzog, C., Dixon, R. A., \& Small, B. J. (1998). Memory change in the aged. New York: Cambridge University Press.

Jacoby, L. L. (1999). Ironic effects of repetition: Measuring age-related differences in memory. Journal of Experimental Psychology: Learning, Memory, Cognition, 25, 3-22. doi: 10.1037/0278-7393.25.1.3

Jacoby, L. L., \& Hay, J. F. (1998). Age-related deficits in memory: Theory and application. In M. A. Conway, S. E. Gathercole, \& C. Cornoldi (Eds.), Theories of memory (pp. 111-134). Hove, UK: Psychology Press. 
Johnson, M. K., Hashtroudi, S., \& Lindsay, D. S. (1993). Source monitoring. Psychological Bulletin, 114, 3-28. doi: 10.1037/0033-2909.114.1.3

Kausler, D. H. (1994). Learning and memory in normal aging. New York: Academic Press.

Kliegl, R., \& Baltes, P. B. (1987). Theory-guided analysis of mechanisms of development and ageing through testing-the-limits and research on expertise. In C. Schooler \& K. W. Schaie (Eds.), Cognitive functioning and social structure over the life course (pp. 95-119). Norwood, NJ: Ablex Publishing.

Kliegl, R., Smith, J., \& Baltes, P. B. (1989). Testing-thelimits and the study of adult age differences in cognitive plasticity of a mnemonic skill. Developmental Psychology, 25, 247-256. doi: 10.1037/00121649.25.2.247

Kliegl, R., Smith, J., \& Baltes, P. B. (1990). On the locus and process of magnification of age differences during mnemonic training. Developmental Psychology, 26, 894-904. doi: 10.1037/0012-1649.26.6.894

Kline, R. B. (2005). Principles and practice of structural equation modeling (2nd ed.). New York: Guilford Press.

Kray, J., \& Lindenberger, U. (2000). Adult age differences in task switching. Psychology and Ageing, 15, 126-147. doi:10.1037/0882-7974.15.1.126

Lehrl, S. (1977). Mehrfachwahl-Wortschatz-Test B $(M W T-B)$. Erlangen, Federal Republic of Germany: Straube.

Lindenberger, U., \& Ghisletta, P. (2009). Cognitive and sensory declines in old age: Gauging the evidence for a common cause. Psychology and Ageing, 24, 116. doi: $10.1037 / \mathrm{a} 0014986$

Lindenberger, U., Nagel, I. E., Chicherio, C., Li, S. C., Heekeren, H. R., \& Backman, L. (2008). Age-related decline in brain resources modulates genetic effects on cognitive functioning. Frontiers of Neuroscience, 2, 234-244. doi: 0.3389/neuro.01.039.2008

Lineweaver, T. T., \& Hertzog, C. (1998). Adults' efficacy and control beliefs regarding memory and aging: Separating general from personal beliefs. Aging Neuropsychology and Cognition, 5, 264-296. doi:10.1076/anec.5.4.264.771

Lo, Y., Mendell, N. R., \& Rubin, D. B. (2001). Testing the number of components in a normal mixture. Biometrika, 88, 767-778. doi: 10.1093/biomet/ 88.3.767

Loken, E., \& Molenaar, P. C. M. (2008). Categories or continua? The correspondence between mixture models and factor models. In G. R. Hancock \& $\mathrm{K}$. M. Samuelsen (Eds.), Advances in latent variable mixture models (pp. 277-297). Charlotte, NC: Information Age Publishing.

Lövdén, M., Bäckman, L., Lindenberger, U., Schaefer, S., \& Schmiedek, F. (2010). A theoretical framework for the study of adult cognitive plasticity. Psychological Bulletin, 136, 659-676. doi: 10.137/a0020080

Lövdén, M., Brehmer, Y., Li, S-C., \& Lindenberger, U. (2012). Training-induced compensation versus magnification of individual differences in memory performance. Frontiers in Human Neuroscience, 6, 141. doi: 10.3389/fnhum.2012.00141

Lövdén, M., Rönnlund, M., Wahlin, A., Backman, L., Nyberg, L., \& Nilsson, L. G. (2004). The extent of stability and change in episodic and semantic memory in old age: Demographic predictors of level and change. Journals of Gerontology: Series $B$ Psychological Sciences, 59(3), P130-134.

Maxwell, S. E., \& Delaney, H. D. (1993). Bivariate median splits and spurious statistical significance. Psychological Bulletin, 113, 181-190. doi: 10.1037/ 0033-2909.113.1.181

Miller, E. K., \& Cohen, J. D. (2001). An integrative theory of prefrontal cortex function. Annual Review of Neuroscience, 24, 167-202. doi: 10.1146/annurev. neuro.24.1.167

Muthén, L. K., \& Muthén, B. O. (1998). Mplus user's guide (6th ed.). Los Angeles, CA: Muthén \& Muthén.

Nagel, I. E., Preuschhof, C., Li, S. C., Nyberg, L., Backman, L., Lindenberger, U. et al. (2009). Performance level modulates adult age differences in brain activation during spatial working memory. Proceedings of the National Academy of Sciences of the United States of America, 106, 22552-22557. doi: 10.1073/pnas.0908238106

Nagel, I. E., Preuschhof, C., Li, S. C., Nyberg, L., Backman, L., Lindenberger, U. et al. (2011). Load modulation of BOLD response and connectivity predicts working memory performance in younger and older adults. Journal of Cognitive Neuroscience, 23, 2030-204. doi: 10.1162/jocn.2010.21560

Nagin, D. S. (1999). Analyzing developmental trajectories: A semi-parametric, group-based approach. Psychological Methods, 4, 139-177. doi: 10.1037/ 1082-989X.4.2.139

Naveh-Benjamin, M., Brav, T. K., \& Levy, O. (2007). The associative memory deficit of older adults: The role of strategy utilisation. Psychology and Aging, 22, 202-208. doi: 10.1037/0882-7974.22.1.202

Naveh-Benjamin, M., Guez, J., Kilb, A., \& Reedy, S. (2004). The associative memory deficit of older adults: Further support using face-name associations. Psychology and Ageing, 19, 541-546. doi: 10.1037/0882-7974.19.3.541

Naveh-Benjamin, M., Shing, Y. L., Kilb, A., WerkleBergner, M., Lindenberger, U., \& Li, S. C. (2009). Adult age differences in memory for name-face associations: The effects of intentional and incidental learning. Memory, 17, 220-232. doi: 10.1080/ 09658210802222183

Noack, H., Lövdén, M., Schmiedek, F., \& Lindenberger, U. (2009). Cognitive plasticity in adulthood and old age: Gauging the generality of cognitive intervention effects. Restorative Neurology and Neuroscience, 27, 435-453. doi: 10.3233/RNN-2009-0496

Oberauer, K. (2003). Understanding serial position curves in short-term recognition and recall. Journal of Memory and Language, 49, 469-483. doi: 10.1016/ S0749-596X(03)00080-9

Old, S. R., \& Naveh-Benjamin, M. (2008). Differential effects of age on item and associative measures of memory: A meta-analysis. Psychology and Ageing, 23, 104-118. doi: 10.1037/0882-7974.23.1.104

Raz, N., Ghisletta, P., Rodrigue, K. M., Kennedy, K. M., \& Lindenberger, U. (2010). Trajectories of brain ageing in middle-aged and older adults: Regional 
and individual differences. Neuroimage, 51, 501-511. doi: 10.1016/j.neuroimage.2010.03.020

Raz, N., Lindenberger, U., Rodrigue, K. M., Kennedy, K. M., Head, D., Williamson, A. et al. (2005). Regional brain changes in ageing healthy adults: General trends, individual differences and modifiers. Cerebral Cortex, 15, 1676-1689. doi: 10.1093/cercor/ bhi044

Roenker, D. L., Brown, S. C., \& Thompson, C. P. (1971). Comparison of measures for estimation of clustering in free recall. Psychological Bulletin, 76, 45-48. doi: 10.1037/h0031355

Rönnlund, M., Nyberg, L., Backman, L., \& Nilsson, L. G. (2005). Stability, growth, and decline in adult life span development of declarative memory: Crosssectional and longitudinal data from a populationbased study. Psychology and Ageing, 20, 3-18. doi: 10.1037/0882-7974.20.1.3

Schneider, W., Kron, V., Hunnerkopf, M., \& Krajewski, K. (2004). The development of young children's memory strategies: First findings from the Würzburg Longitudinal Memory Study. Journal of Experimental Child Psychology, 88, 193-209. doi: 10.1016/ j.jecp.2004.02.004

Schneider-Garces, N. J., Gordon, B. A., BrumbackPeltz, C. R., Shin, E., Lee, Y., Sutton, B. P. et al. (2010). Span, CRUNCH, and beyond: Working memory capacity and the ageing brain. Journal of Cognitive Neuroscience, 22, 655-669. doi: 10.1162/ jocn.2009.21230

Schwartz, G. (1978). Estimating the dimension of a model. The Annals of Statistics 4, 461-464. doi: 10.1214/aos/1176344136

Shing, Y. L., Werkle-Bergner, M., Brehmer, Y., Muller, V., Li, S. C., \& Lindenberger, U. (2010). Episodic memory across the lifespan: The contributions of associative and strategic components. Neuroscience and Biobehavioral Reviews, 34(7), 1080-1091. doi: 10.1016/j.neubiorev.2009.11.002

Shing, Y. L., Werkle-Bergner, M., Li, S. C., \& Lindenberger, U. (2008). Associative and strategic components of episodic memory: A life-span dissociation. Journal of Experimental Psychology: General, 137, 495-513. doi: 10.1037/0096-3445. 137.3.495
Shing, Y. L., Werkle-Bergner, M., Li, S. C., \& Lindenberger, U. (2009). Committing memory errors with high confidence: Older adults do but children don't. Memory, 17, 169-179. doi: 10.1080/ 09658210802190596

Simons, J. S., \& Spiers, H. J. (2003). Prefrontal and medial temporal lobe interactions in long-term memory. Nature Reviews Neuroscience, 4, 637-648. doi: 10.1038/nrn1178

Singer, T., Verhaeghen, P., Ghisletta, P., Lindenberger, U., \& Baltes, P. B. (2003). The fate of cognition in very old age: Six-year longitudinal findings in the Berlin Ageing Study (BASE). Psychology and Ageing, 18, 318-331. doi: 10.1037/0882-7974.18.2.318

Snodgrass, J. G., \& Corwin, J. (1988). Pragmatics of measuring recognition memory: Applications to dementia and amnesia. Journal of Experimental Psychology-General, 117, 34-50. doi:10.1037/00963445.117.1.34

Spencer, W. D., \& Raz, N. (1995). Differential effects of ageing on memory for content and context: A metaanalysis. Psychology and Ageing, 10, 527-539. doi: 10.1037/0882-7974.10.4.527

Tabachnik, B. C., \& Fidell, L. S. (2006). Cleaning up your act: Screening data prior to analysis. In B. C. Tabachnik \& L. S. Fidell (Eds.), Using multivariate statistics (5th ed., pp. 61-116). Needham Heights, MA: Allyn \& Bacon.

Tulving, E. (1972). Episodic and semantic memory. In E. Tulving \& W. Donaldson (Eds.), Organisation of memory (pp. 381-403). New York: Academic Press.

Verhaeghen, P., \& Marcoen, A. (1996). On the mechanisms of plasticity in young and older adults after instruction in the method of loci: Evidence for an amplification model. Psychology and Ageing, 11, 164-178. doi: 10.1037/0882-7974.11.1.164

Verhaeghen, P., Marcoen, A., \& Goossens, L. (1992). Improving memory performance in the aged through mnemonic training: A meta-analytic study. Psychology and Ageing, 7, 242-251. doi: 10.1037/ 0882-7974.7.2.242

Wechsler, D. (1955). Wechsler Adult Intelligence Scale manual. New York: Psychological Corporation. 